COMMENT. The prevalence of multiple sclerosis (MS) has increased over the past half century. A study in Norway found the incidence by year of onset increased 2-fold from 2.87/100,000 in 1950-54 to 5.57/100,000 in 1985-91.(Midgard R et al. Brain 1996;119:203211). The increase was a general trend and was not explained by a change in age distribution. Interest in childhood onset MS has increased and several reports have appeared in the literature in the past decade, the most recent from Taiwan of 21 cases. (Weng W-C et al. Pediatr Neurol 2006;35:327-334)(AAP Grand Rounds 2007;17:30-31). A female-to-male ratio of $2.5: 1$ in Taiwanese children is greater than that in the above multinational study (1.54:1) and closer to that of a Canadian collaborative study of 3.2:1. (Orton S-M et al. Lancet Neurol 2006;5:932-936). The female preponderance for MS has increased in the past 50 years, and daughters of female MS patients have a 50-fold increase in risk of contracting MS. (Sadovnick AD et al. Neurology 1988;38:990-991). Heritable risk factors for MS, recently identified by a genomewide study, include alleles of interleuken-2-receptor gene $(I L 2 R A)$ and $I L 7 R A$.(Hafler DA, et al. N Engl J Med August 30, 2007;357:851-862)

MS is usually considered an autoimmune disease, but the mechanism is unproven. Recent work supports a viral etiology, with Ebstein-Barr virus having an important role in the initiation and progression of symptomatic autoimmunity. (Lunemann JD et al. Curr Neurol Neurosci Rep 2007;7:253-258)(Lipton HL et al. Ann Neurol 2007;61:514-523). Human herpesvirus-6, not included in the multinational study, may play a lesser role triggering attacks in relapsing-remitting MS. (Alvarez-Lafuente R et al. Mult Scler 2007;13:578-583). Noninfectious factors sometimes implicated in the etiology of MS include geographic, environmental determinants, and the change in risk among migrants. Cigarette smoking may contribute to the increases reported in the female/male ratio and MS incidence. Sunlight exposure and vitamin D may have a protective effect.(Ascherio A et al. Ann Neurol 2007;61:504-513). Clinical evidence in support of viral infection in etiology include the viral prodrome, usually upper respiratory in type, that occurs in approximately one half of childhood onset MS cases, and oligoclonal bands in the CSF.

\title{
DISSEMINATED ENCEPHALOMYELITIS AND MULTIPLE SCLEROSIS DIFFERENTIATION
}

The distinguishing features of acute disseminated encephalomyelitis (DEM) and multiple sclerosis (MS) are reviewed by researchers at Harvard Medical School, Boston, MA, and University of Zagreb, Croatia. Acute and recurrent DEM affect children more than adults. Symptoms of DEM such as fever, altered consciousness, aphasia, and meningism are rare in MS. CSF oligoclonal bands are also rare. Genetic factors are often involved in MS but they are absent in ADEM. MRI is the best method of distinguishing the two distinct diseases. In DEM or ADEM, the lesions are larger than the plaques found in MS, they enhance with gadolinium, and often involve the thalamus and basal ganglia. MRI McDonald criteria for the diagnosis of MS require one enhancing or nine T-2 lesions: one infratentorial and one juxtacortical; and three or more periventricular lesions. The demyelinating lesions are disseminated in both diseases but differ in form and size; in MS the plaques are small and have characteristic sharply defined borders, whereas in ADEM they are large and ill-defined, inflammatory and perivenous.

Both brain and spinal cord MRI should be obtained in cases of clinically isolated syndrome (CIS) suggesting demyelination. Spinal cord lesions in DEM are elongated, 
whereas in MS they are less than two or three vertebral segments in length. In ADEM, cerebral MRI lesions may be massive, sometimes mistaken for tumors, Schilder's or Balo's disease, or strokes. Devic's syndrome or neuromyelitis optica, called 'Oriental form of MS' in Japan, has been classified as a form of MS in the past. Recent reports suggest that Devic's syndrome is different from MS and more closely resembles DEM. Treatment and prognostic considerations are important reasons to differentiate between a CIS of MS and acute DEM, and between relapsing-remitting MS and recurrent DEM. Lifelong immunomodulatory treatment is initiated immediately after an MS CIS, whereas IV methylprednisolone, IV immunoglobulin G, or plasma exchange are accepted treatments for ADEM. (Poser CM, Brinar VV. Disseminated encephalomyelitis and multiple sclerosis: two different diseases - a critical review. Acta Neurol Scand October 2007;116:201-206). (Respond: Charles M Poser MD, 11 Rutland Square, Boston, MA o2118).

COMMENT. The differentiation of ADEM and MS is made on clinical history, neurologic and MRI findings, and CSF analysis. Given the variety and remitting nature of clinical symptoms of demyelinating disease, a neurologist with expertise in neuroradiology may be best qualified to correctly diagnose the disorder. The above review regards the two diseases as distinct entities and not parts of a spectrum, as sometimes proposed.

The spectrum of neuromyelitis optica, (NMO). Researchers from the Mayo Clinic, Scottsdale, AZ, and Rochester, $\mathrm{MN}$, review the role of the serum autoantibody marker (NMO-IgG) in the pathogenesis of NMO and in the definition of an extended spectrum of NMO-related disorders, distinct from MS. (Wingerchuk DM et al. Lancet Neurol September 2007;6:805-815). The potential pathogenicity of NMO-IgG, the role of aquaporin 4 as the inducer and target of the autoimmune attack, with resulting inflammatory demyelination and necrosis, and treatment are discussed. The revised Wingerchuk diagnostic criteria for NMO require 2 of the following: 1) Brain MRI is not diagnostic of MS; 2) spinal cord lesion must be 3 or more segments in length; and 3) patient is NMO-IgG antibody seropositive.

NMO in a mother and daughter is reported from University of Michigan, Ann Arbor. (Braley T, Mikol DD. Arch Neurol August 2007;64:1189-1192). The ages of onset were 62 and 29 years, respectively. Few familial cases of NMO are described.

\section{SEIZURE DISORDERS}

\section{MORTALITY OF STATUS EPILEPTICUS IN ALL AGE GROUPS}

The in-hospital mortality and potential predictors of outcome of generalized convulsive status epilepticus (GCSE) were evaluated at the Neurological Institute, Case School of Medicine, Cleveland, $\mathrm{OH}$. The cohort was identified from the Nationwide Inpatient Sample (NIS) databases for the years 2000 through 2004. The definition of status epilepticus employed was continuous seizures or repetitive seizures without recovery of consciousness of 30 or more minutes' duration. The analysis included 11,580 patients (mean age $39+/-25.6$ years, $53.4 \%$ male, $42.4 \%$ white) with a median hospital stay of 3 days. Mortality was $3.45 \%$ overall. Adjusted mortality rates by age were $0.67 \%$ for patients $<$ or $=10$ years, $1.33 \%$ for ages $11-20$, increasing to $10.15 \%$ for patients $>80$ years. Potential 